\title{
Rolling Element Bearing Faults Detection, Wavelet De-noising Analysis
}

\author{
Maamar Ali Saud AL-Tobi ${ }^{1, *}$, Khalid F. Al-Raheem ${ }^{2}$ \\ ${ }^{1}$ Caledonian College of Engineering-Oman, Sultanate of Oman \\ ${ }^{2}$ College of Engineering, Sohar University, Sultanate of Oman
}

Copyright (C) 2015 Horizon Research Publishing All rights reserved.

\begin{abstract}
The wavelet de-noising technique with wavelet based function has been used in this paper for bearing fault detection. The applications of the wavelet de-noising show that the fault pulses in time-domain of the de-noised signals are easily to be detected as a result of removing the covering noise, which is not possible through the time-domain analysis of the original signal. Furthermore, the reciprocal period which matches the bearing fault frequency can be easily detected without further analysis by FFT-Spectrum.
\end{abstract}

Keywords Wavelet Transform, Wavelet De-noising, Morlet, Bearing Fault Detection

\section{Introduction}

The task of improvement begins with the progress towards an advanced life that the industries and production organizations become more needful for ideal products that must be given in standard time with so many other extreme requirements. With these present necessary conditions, the necessity to create a balance between the extreme consumption of machines throughout the frequent demands for the products and the effects of frequent failures in the machines can lead to expensive replacement costs incurred However, if it is neglected there can be a cause of a catastrophic accident that may lead to production downtime potential failure in the supply. The continuous based maintenance provides the effective solutions to minimize the consequences of machine failures that have obtained to give preventive maintenance through the predictive maintenance. CBM is used to give an affirmation for the long use of machines elements. Condition monitoring is very essential part for manufacturing. This provides longer machinery operating at the least overall cost. All that will accomplish the purpose of creating a balance in the conflict between the desire to produce many products and at the same time to provide a longer machinery operation. One of the common widely used elements is rolling element bearing that were developed in order to eliminate sliding friction. Most of the industrial machines are observed to use this type of element frequently, which has led to some researches on this bearing to detect the fault. The bearing is often found to be primary responsible for the machine breakdown. On other hand, the reasons which lead to the failure of the bearing can be presented in different forms such as a machine running unbalanced, misaligned, at a critical speed; a bearing fitted incorrectly; the wrong grease being used; or maybe no grease being used at all. The most common machinery vibration problems are caused by unbalance or misalignment, which leads to the bearing failure (Howieson, 2003) [4]. As a good way to enter the concept of wavelet transform is to understand the concept of time-frequency analysis. It has been known that the signal can be analyzed in one way whether in time or frequency domains and that is only suitable for the stationary signals. But, using these concepts is unsuitable for the non-stationary signals which require both the information of time and frequency, so the reasons are that frequency domain analysis gives averaged spectral coefficients, which represent the frequency contents of a random process and independence of time. (Politis, et al.2005) [6]. The time domain analysis on the other hand, gives no information at all about the frequency content of the signal (Fyfe, et al. 2004) [3]. Moreover, wavelet is a powerful technique that provides multi-resolution in time and frequency domain (Bentley and McDonnell, 1994) [2]. In a research for (AL-Raheem, et al. 2006) [1] presented results of effects of using wavelet transform to detect the rolling element bearing faults. The results show the effectiveness of the used wavelet de-noising technique to extract the fault impulses and its period, and its sensitivity to the variation of the fault frequency as a result of changing the shaft rotational speed. Also, in the same research (AL-Raheem, et al. 2006) [1] showed the results of using Laplace-Wavelet Envelope Power Spectrum to diagnosis rolling element bearing faults. There has shown effectiveness of Laplace Wavelet Envelope Power Spectrum in the extraction of the BCF and its harmonics, for outer and inner race defective bearings from noisy vibration signals. As a requirement to use wavelet analysis effectively, the wavelet de-noising is presented which is used to remove the 
unwanted coefficients which are as a result of signal decomposition using wavelet transform. In this case the desire for many thresholding to remove these coefficients. This technique is supported by three steps which decompose the signal by using wavelet transform, then to remove the coefficients which are produced after the decomposition and finally reconstruct the signal from threshold wavelet coefficients using inverse wavelet transform (Fyfe, et al. 2004) [3].

In this study the wavelet de-nosing is employed to detect the rolling element bearing faults using morlet as a selected wavelet function.

This paper is organized as follows. In the next section, theory of Wavelet analysis will be discussed. Then in section 3 , the experimental set up followed by section 4 which discuss the results. Finally, conclusions are presented in section 5 .

\section{Theory}

As a first introduction of wavelet was presented by Goupillaud (primer, 1998) [6] and that was for seismic records analysis. The parameter a, corresponds to a dilation or compression of the mother wavelet which is in this case defined as scale. The second parameter, b, identify a shift of the mother wavelet along the $\mathrm{x}$-domain. In the case of wavelet transform, the frequency variable $\omega$ is replaced by the scale variable a and generally the time-shift variable $\tau$ is represented by $b$. Hence, the wavelet is represented as (Sheng, 2000) [8]:

$$
\psi_{\mathrm{b}, \mathrm{a}}(\mathrm{t})=\frac{1}{\sqrt{\mathrm{a}}} \mathrm{h}^{*}\left(\frac{\mathrm{t}-\mathrm{b}}{\mathrm{a}}\right)
$$

The continuous wavelet transform (CWT) is represented as:

$$
\begin{gathered}
\mathrm{WT}\{\mathrm{x}(\mathrm{t}), \mathrm{a}, \mathrm{b}\}=<\Psi_{\mathrm{a}, \mathrm{b}} \cdot \mathrm{x}(\mathrm{t})> \\
\mathrm{s}=\frac{1}{\sqrt{\mathrm{a}}} \int \mathrm{x}(\mathrm{t}) \Psi^{*}{ }_{\mathrm{a}, \mathrm{b}}(\mathrm{t}) \mathrm{dt}
\end{gathered}
$$

Based on this equation, it is clear that the WT performed a decomposition of the signal. Where $<>$ points out to the inner product. The $\Psi$ (a,b )is a family of wavelet. Figure 1 demonstrates the machinery of wavelet functions which works for different values of the scale parameter a. consequently, the scaling makes the wavelet functions express the same number of cycles within the support of the mother wavelet.
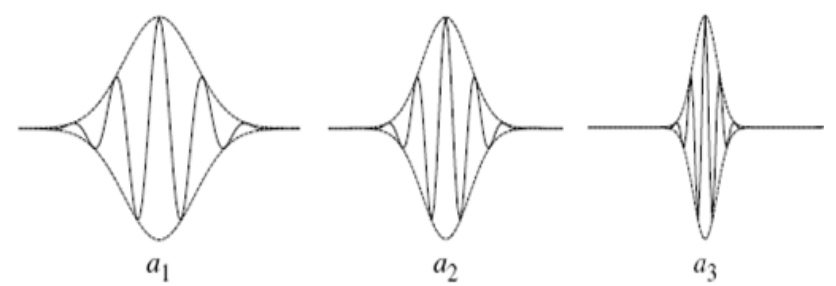

Figure 1. The machinery of wavelet functions (Politis et al, 2005)

\subsection{Wavelet De-noising Technique}

The first step is the continues wavelet transform which decomposes the signal and it is defined in Equation 2.

The second step is thresholding that it works to shrink the wavelet coefficients and it is defined as:

$$
\text { thr }=\sigma \sqrt{2} \log N
$$

Where $\mathrm{N}$ is the number of data samples in the signal and $\sigma$ is the scale parameter.

The third step is inverse continues wavelet transform ( ICWT) and it is represented as:

$$
x(t)=C_{\Psi}-1 \int_{-\infty}^{\infty} W(a, t) \frac{d a}{a^{\frac{3}{2}}}
$$

Where,

$$
C_{\Psi}=\int_{-\infty}^{\infty} \frac{\Psi^{\wedge *}(\omega)}{|\omega|} d \omega
$$

\subsection{Morlet}

Morlet is the selected wavelet-function as it has more similarity with the extracted fault pulses shape and it is represented as

$$
\Psi(\mathrm{x})=\mathrm{Ce}^{-\mathrm{x}^{2} / 2} \cos (5 \mathrm{x})
$$

The constant $\mathrm{C}$ is used for normalization in view of reconstruction.

Figure 2 indicates to this function

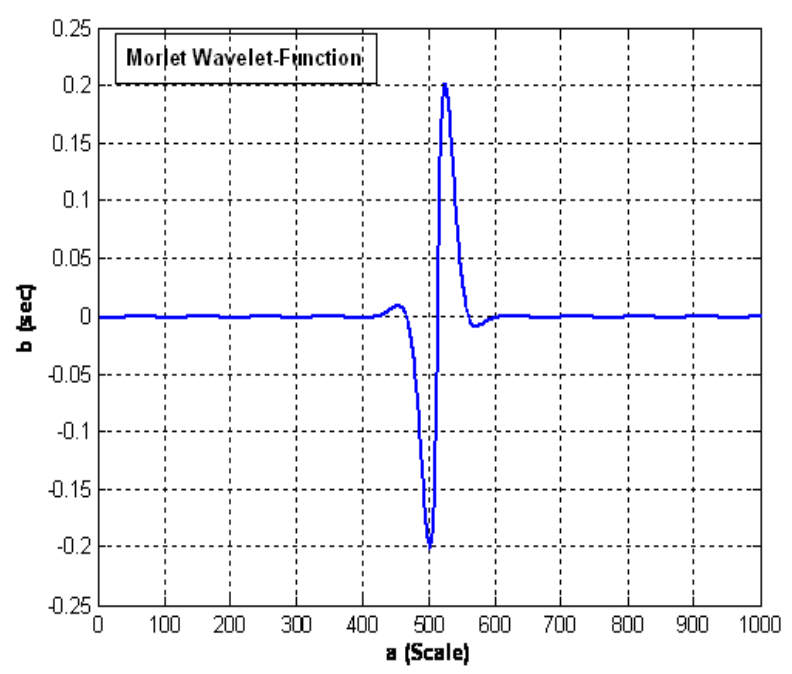

Figure 2. Morlet Wavelet-function

\section{Experimental Set Up}

This experiment was conducted in the fatigue and vibration laboratory in the college of engineering in Sultan Qaboos University (SQU) using Machine Fault Simulator (MFS). The used sensor is accelerometer sensor. A PC with a data acquisition card and LabView software were used. 


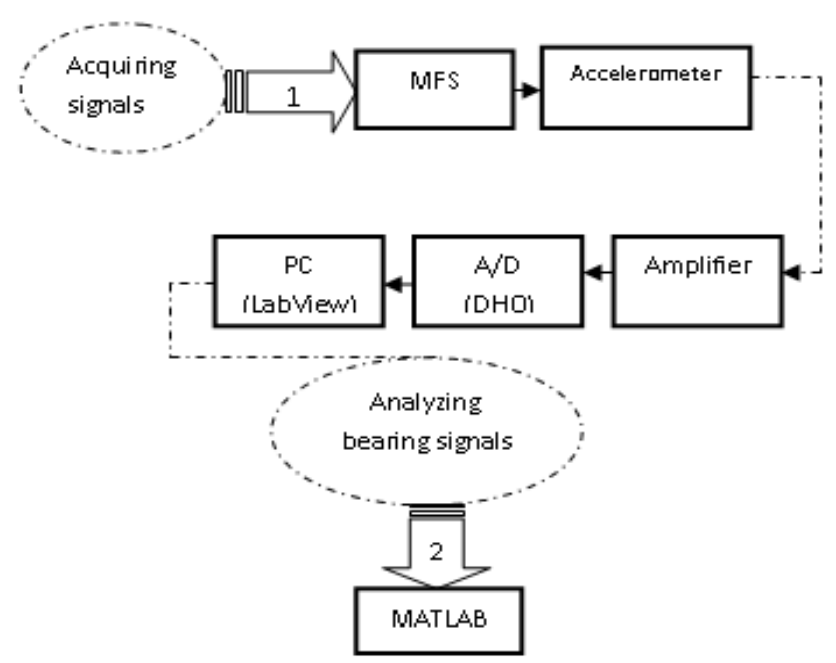

Figure 3. The experimental set-up

Figure 3 shows the schematic diagram of the experimental setup. The shaft is supported by two rolling element bearings. The machine is driven by a $0.5 \mathrm{hp}$ three phase AC motor. The speed of the motor can be varied using electronic speed controller in the range of 0-10,000 RPM. Machine loads can also be varied using magnetic break, which is manually adjustable in the range of 0.5-10 lb-in. A triggering signal was produced using Bently Nevada proximity sensor to start the measurement from the same referenced point. The signals were acquired through a PC with a data acquisition card (DAQ) from National Instruments (type AT-MIO-16XE52 10). The card has 16 single-ended or 8 differential channels with a resolution of 16 bits.

The DAQ card has a low pass anti-aliasing filter with selectable cutoff frequency in the range of $0.25 \mathrm{kHz}$. A Lab View virtual instrument was developed to acquire the bearing signals. Experiments were conducted using MB 204 sealed rolling element bearings with shaft centering feature. Two conditions of bearings were used: healthy (Normal) and faulty bearings. For the present work, the following values have been used: $\mathrm{SRF}=10 \mathrm{~Hz}, 20 \mathrm{~Hz}$ and $30 \mathrm{~Hz}, \mathrm{~d}=7.14 \mathrm{~mm}$, $\mathrm{D}=28.519 \mathrm{~mm}, \alpha=3.7404^{\circ}$ and $\mathrm{n}=8$. Where SRF is the shaft rotation frequency, $d$ is the rolling element diameter, $D$ is the pitch circle diameter, $\alpha$ is the contact angle and $n$ is the number of rolling elements.

The collection of the vibration signals were obtained using sampling rate (fs) of $12000 \mathrm{~Hz}$ with 121000 samples and the recording time of 10.08 second.

\section{Results}

The characteristic fault frequencies of the rolling element bearings are calculated according to the formulae are included in the appendix. Then, the faults on the outer faulty bearing, inner faulty bearing and roller faulty bearing can be detected according to the given characteristic fault frequencies (Mitchell, 1993) [5]. The extracted data are based on four different shaft rotational speeds which they are $10 \mathrm{~Hz}, 20 \mathrm{~Hz}, 25 \mathrm{~Hz}$ and $30 \mathrm{~Hz}$.
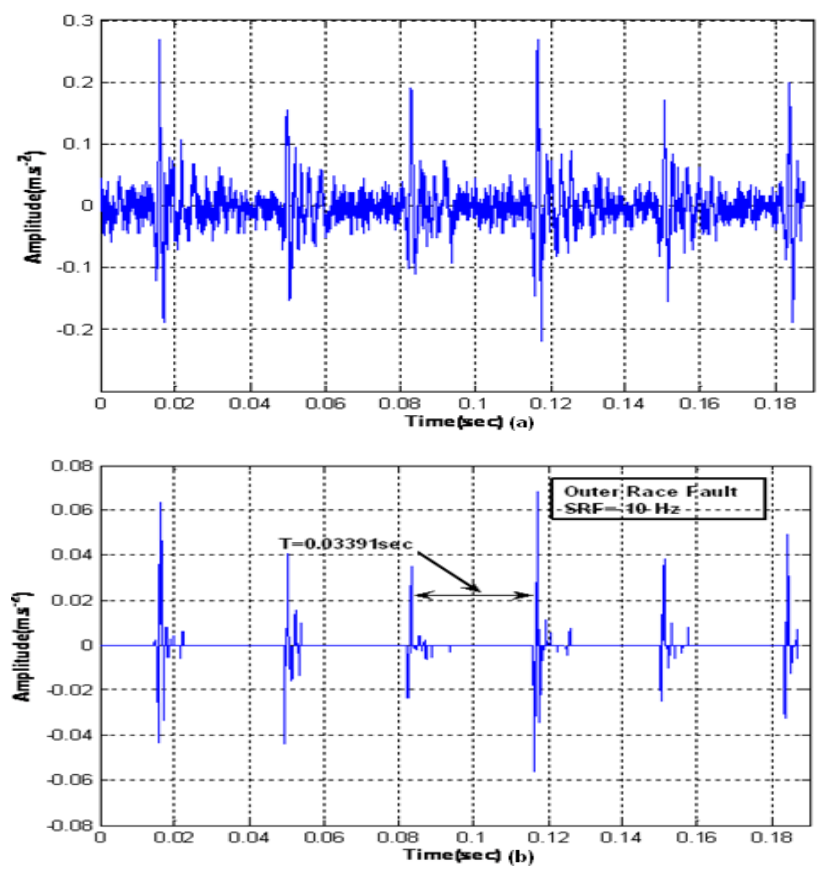

Figure 4. The outer race fault at $10 \mathrm{~Hz}$ (a) the measured bearing vibration signal (b) the de-noised vibration signal
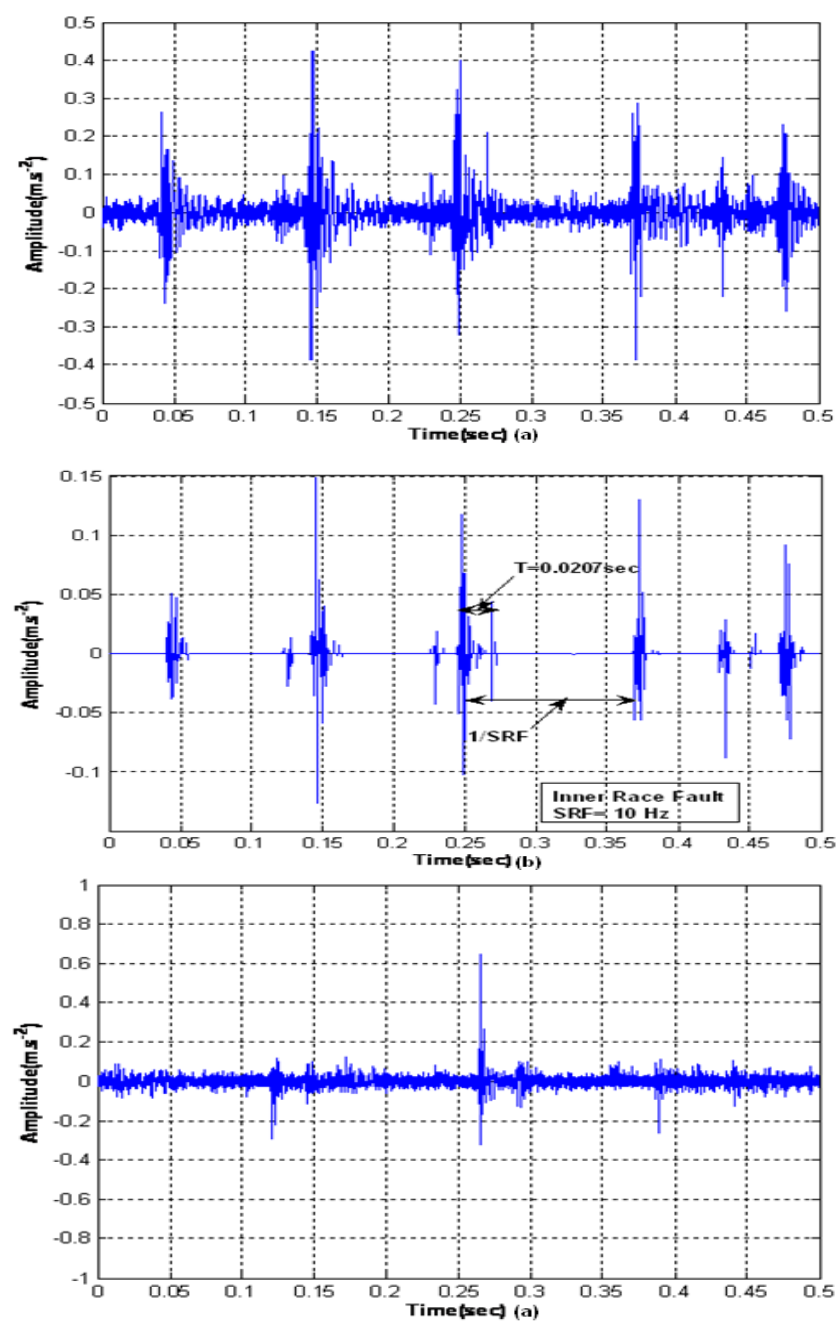

Figure 5. The inner race fault at $10 \mathrm{~Hz}$ (a) the measured bearing vibration signal (b) the de-noised vibration signal. 


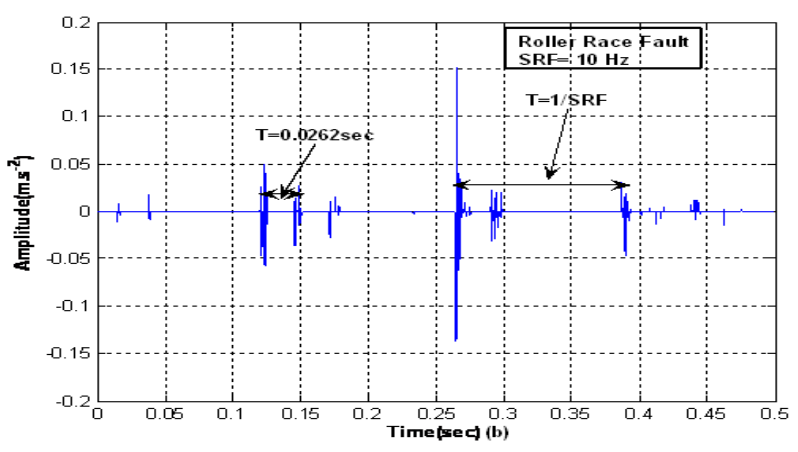

Figure 6. The Roller race fault at $10 \mathrm{~Hz}$ (a) the measured bearing vibration signal (b) the de-noised vibration signal

\subsection{Outer Race Fault}

The case of outer race fault can be considered as the easiest suitable, effective and analytical way as it is being a stationary. Therefore, the impulses are continual and as well as the amplitude is approximately equal and that is observed through the equal distance between each impulse. Figure 4 shows both the signal of outer race fault before wavelet de-noising and impulses of outer race fault after wavelet de-noising. In Figure 4 (a), it is noticed that it is a noisy signal, but in Figure 4 (b), it shows no noise to being de-noised. As a result, Table 1 shows the values of calculated and extracted frequencies for the outer race fault with different shaft rotational speeds. These values are plotted in Figure 7.

Table 1. The calculated and extracted BPF

\begin{tabular}{|c|c|c|}
\hline $\begin{array}{c}\text { SRF } \\
(\mathrm{Hz})\end{array}$ & $\begin{array}{c}\text { BPF } \\
\text { Calculated } \\
(\mathrm{Hz})\end{array}$ & BPF Extracted $(\mathrm{Hz})$ \\
\hline 10 & 30.0070 & 29.48 \\
\hline 15 & 45.0104 & 45.59 \\
\hline 20 & 60.0139 & 59.70 \\
\hline 25 & 75.0174 & 76.21 \\
\hline 30 & 90.0209 & 89.92 \\
\hline
\end{tabular}

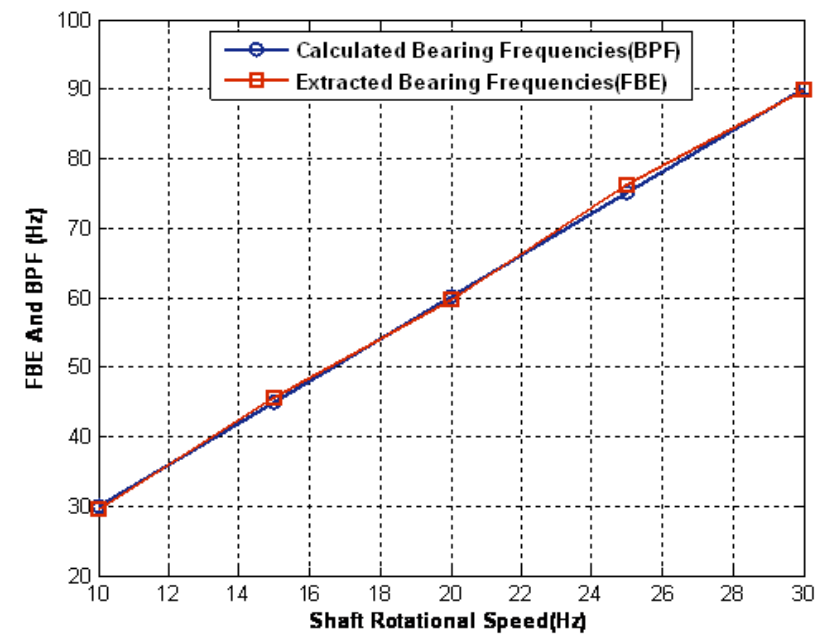

Figure 7. The values of calculated and extracted frequencies of outer race fault

\subsection{Inner Race Fault}

The inner race fault is unlike the outer race fault that it is rotating with the shaft and the appearance of fault concentrates with the area of load zone, hence, in this cause the impulses become difficult to diagnose and with the increasing of shaft rotational speed, the impulses become more difficult. As a consequence, the amplitude is noticed to be not equal. From Figure 5 (a) and (b) which indicates to the measured bearing vibration signal at $10 \mathrm{~Hz}$ (i.e. before wavelet de-noising), it shows clearly the period between each impulse. From the same figure below, the passage of the inner race into and out of the load zone with shaft rotation produces an amplitude variation of the inner race fault frequency. The resulting modulation will produce sidebands at shaft rotating frequency.

\subsection{Roller Race Fault}

The roller race fault is similar to the inner race fault except that it rotates twice, once with the shaft along with the inner and once inside the bearing. From figure 5, it is obvious that the first small impulses are away from the load zone but the biggest one indicates to the area of center load zone. The period between each impulse is the inverse of Shaft Rotational Speed (SRF). Figure 6 (a) points out to the measured bearing vibration signal and Figure 6 (b) the de-noised vibration signal. E.g. Peter [10] found an innovative scientific phenomenon.

\section{Conclusions}

The results of using wavelet de-noising technique can be concluded as the followings:

- The fault pulses in Time-Domain are easy to detect through removing the covering noise (de-noising) which is not possible through Time-Domain analysis.

- The period reciprocal BPF which matches the bearing fault frequency can be easily calculated without need for further analysis by FFT-Spectrum.

- The effectiveness of the Wavelet De-noising can be improved by selecting a wavelet-function with more similarity with the fault pulses shape.

\section{REFERENCES}

[1] K.F. Al-Raheem,. Detection of the rolling element bearing faults using optimized- wavelet de-noising technique. , 8th Int. Conf. on Signal Processing, ICSP'06 Proceedings, pp.2978-2981, 2006.

[2] P.M Bentley. And J.T.E McDonnell. Wavelet transforms: an introduction. Electronics and communication engineering journal. IEE conf. publ. 387, 1994. 
[3] K.R. Fyfe, J Lin,. and M. J. Zuo Mechanical fault detection based on the wavelet de-noising technique", ASME. J. of vibration and Acoustics, vol. 12(6), pp. 9-16, 2004.

[4] D. Howieson. Vibration Monitoring: Envelope Signal Processing. Diagnostic instruments, SKF Reliability Systems, 2003.

[5] J. Mitchell. Introduction to machinery analysis and monitoring, 2nd edition, Penn Well Publishing Company 1421 South Sheridan/P.O. Box 1260 Tulsa, Oklahoma 74101, 1993.
[6] N.P. Politis, P.D. Spanos,. and G. Failla. Wavelets Concepts and Applications. Taylor \& Francis Group, LLC. Kevin Englehart, 2005.

[7] A. Primer, C.S. Burrus, R.A.Gopinath, and H. Guo,. Introduction to Wavelets and Wavelet Transforms. By Prentice- Hall, Inc. Upper Saddle River, New Jersey 07458, 1998.

[8] Y. Sheng. Wavelet Transform. The Transforms and Applications Handbook, Second Edition. Ed. Alexander D. Poularikas Boca Raton: CRC Press LLC, 2000 